\title{
Citogenética y citoquímica de pacientes con leucemia en dos hospitales neotropicales
}

\author{
María Virginia Solís ${ }^{1}$, María de los Angeles Alvarado ${ }^{2}$, Edward Ruiz ${ }^{1}$, Juan Manuel Carrillo \\ Marta Navarrete ${ }^{3}$, German Sánchez ${ }^{4}$ y Elías Jiménez ${ }^{3}$. \\ 1 Escuela de Biología, Universidad de Costa Rica, 2060 San José, Costa Rica. Fax (506) 207-4216 \\ msolis@biologia.ucr.ac.cr. \\ 2 Facultad de Microbiología, C.I.A.T.A., Universidad de Costa Rica, 2060 San José, Costa Rica. \\ 3 Hospital Nacional de Niños, Hematología, San José, Costa Rica. 4Hospital Calderón Guardia, \\ Hematología, San José, Costa Rica.
}

Recibido 31-VIII-1999. Corregido 15-II-2000. Aceptado 24-II-2000.

\begin{abstract}
A cytogenetic and/or cytochemical study was performed in 166 individuals with leukemia or related disorders, in two major Costa Rican hospitals. In those patients treated at an adult's hospital (14 years old and over), acute leukemias represented $66 \%$ of all cases. In that hospital the most frequent types of disorders were, in decreasing order: ANLL (>M1), ALL, CML (all of them showed the Ph chromosome) and MDS. In the cases from a childrens' hospital ( $<14$ years old) acute leukemias were $98 \%$. Among them the order of frequency was: ALL (70\%): ALL-1 (84\%), ALL-2(16\%) and ANLL (27\%): M5a $>$ M3>M4>M5b. In ALL 85\% were type B and occurred mostly in women while $15 \%$ of them were type $\mathrm{T}$ and more frequent in males. There was $5.6 \%$ infant leukemia, which presented a similar number of acute lymphoids and myeloids. The cytogenetic pattern was similar among Costa Rica and other tropical and temperate countries.
\end{abstract}

Key words: Leukemia cytogenetics, acute lymphocytic leukemia, acute nonlymphocytic leukemia, chronic granulocytic leukemia.

Los estudios cromosómicos de las leucemias humanas durante las últimas tres décadas, han dado un gran aporte al conocimiento de los cambios genéticos que ocurren en estas enfermedades.

En los trastornos hematológicos malignos, se detectan anormalidades cromosómicas recurrentes, varias de las cuales correlacionan con subtipos de leucemia que tienen rasgos clínicos, morfológicos e inmunológicos característicos, tales como la respuesta a la terapia (Anónimo 1981). Por ejemplo, en la leucemia aguda linfocítica en niños, el grado de ploidía y la presencia o ausencia de translocaciones específicas, son considerados como factores pronósticos importantes e independientes (Pui et al. 1990).
El estudio de las anormalidades cromosómicas en las leucemias cumple varias funciones. Contribuye a establecer un diagnóstico más preciso. Proporciona información pronóstica y permite una selección más racional de la terapia para un paciente en particular (Le Beau 1991). Además hace posible identificar los sitios que presentan rearreglos consistentes, los cuales pueden ser luego aislados y analizados molecularmente (Le Beau 1991).

El objetivo principal del trabajo presente fue realizar una caracterización citoquímica y citogenética de varios pacientes con leucemia y otros trastornos hematológicos relacionados, de los hospitales Nacional de Niños y Dr. Rafael Angel Calderón Guardia. 


\section{MATERIALES Y MÉTODOS}

Desde mayo de 1993 y hasta diciembre de 1996, se realizaron estudios citogenéticos y / o citoquímicos a 107 pacientes del Hospital Nacional de Niños (HNN) y a 49 del Hospital Dr. Rafael Angel Calderón Guardia (HCG). Todos los niños fueron casos nuevos. Los adultos fueron casos nuevos o pacientes que no se encontraban en tratamiento en ese momento. Se seleccionaron de manera consecutiva.

A. Estudios citogenéticos. De cada paciente se obtuvieron las siguientes muestras: 3 $\mathrm{ml}$ de sangre periférica (muestra uno), $0.5-1 \mathrm{ml}$ de médula ósea (muestra dos), 0.2-0.5 $\mathrm{ml} \mathrm{de}$ médula ósea en $10 \mathrm{ml}$ de medio de transporte (muestra tres) y $1 \mathrm{ml}$ de sangre periférica en 10 $\mathrm{ml}$ de medio de transporte (muestra cuatro, únicamente en los niños). El medio de cultivo de transporte consistía de un medio completo al cual se le agregó $10 \mu \mathrm{g} / \mathrm{ml}$ de bromuro de etidio, $0.01 \mu \mathrm{g} / \mathrm{ml}$ colcemid y $20 \mathrm{U} / \mathrm{ml}$ heparina de sodio (Modificado de Williams et al. 1984).

En todos los tipos de leucemia, con excepción de la leucemia crónica linfocítica, se aplicaron las siguientes cinco técnicas a cada paciente:

1. Obtención directa de metafases de médula ósea. En este caso se utilizó la muestra tres. Se dividió el contenido del tubo en dos partes y a cada una de ellas se le realizaron dos lavados con medio RPMI-1640. 2. Obtención directa de metafases de sangre periférica. $\mathrm{Se}$ realizó usando la muestra cuatro. 3 . Cultivo de 24 horas de médula ósea. Se inoculó 0.1-0.2 $\mathrm{ml}$ de la muestra dos, en cada uno de tres tubos con $10 \mathrm{ml}$ de medio de cultivo completo con la siguiente composición : $100 \mathrm{ml}$ de medio RPMI-1640, $20 \mathrm{ml}$ de suero bovino fetal, $1 \mathrm{ml}$ penicilina/estreptomicina, $1 \mathrm{ml} \mathrm{L}$-glutamina, $0.2 \mathrm{~g} \mathrm{NaHCO}_{3}, 20 \mathrm{U} / \mathrm{ml}$ heparina de sodio (Williams et al. 1984). Los tubos se incubaron por 24 horas a $37^{\circ} \mathrm{C}$. Hora y media antes de cumplirse dicho tiempo las células se expusieron a un tratamiento con colcemid $(0.01 \mu \mathrm{g} / \mathrm{ml})$ y bromuro de etidio $(10 \mu \mathrm{g} / \mathrm{ml})$. 4. Cultivo de 24 horas de sangre periférica. Se inoculó $1 \mathrm{ml}$ de la muestra uno en cada uno de dos tubos con 10 $\mathrm{ml}$ de medio de cultivo completo. Se cultivaron por 24 horas a $37^{\circ} \mathrm{C}$. En todos los casos anterio- res se hizo un choque hipotónico con una solución de $\mathrm{KCl} 0.075 \mathrm{M}$ a $37^{\circ} \mathrm{C}$ por 35 minutos incluyendo la centrifugación y cuatro a seis fijaciones de 30 minutos cada una con tres partes de metanol y una de ácido acético. 5. Cultivo por $\mathbf{7 2}$ horas de sangre periférica. Se inoculó $0.5 \mathrm{ml}$ de la muestra uno en dos tubos con medio completo y fitohemaglutinina, según la técnica de Rooney \& Czepulkowski 1986. El propósito fue realizar un cultivo de control. En la leucemia crónica linfocítica se utilizó el mismo medio de cultivo anterior, se agregó lipopolisacárido de $E$. coli, a una concentración de 20 $\mu \mathrm{g} / \mathrm{ml}$ y se incubó por 72 horas a $37^{\circ} \mathrm{C}$.

En todos los procedimientos descritos, se hicieron las láminas lanzando el sedimento celular desde una altura de $95 \mathrm{~cm}$. Luego se pasaron ligeramente por la llama de una lámpara de alcohol. Cinco láminas del cultivo directo y corto de médula ósea fueron teñidas con Giemsa, para realizar el conteo cromosómico en 3050 células y determinar el número modal (NM)( número más frecuente de cromosomas). El resto fueron bandeadas, utilizando una modificación del metódo de Seabright 1971. Se disolvió la tripsina en un medio mínimo (Minimum Essential Medium Eagle), a pH de 7.27.4 corregido con $\mathrm{NaHCO}_{3}$, al $5.5 \%$ y se tiñeron con Giemsa a pH 6.8. Los cariotipos fueron descritos utilizando la nomenclatura internacional (Anónimo 1992).

B. Estudios citoquímicos (Dacie y Lewis 1987, Scott et al.1993). Se emplearon muestras de sangre periférica con EDTA o frotis de aspirado de médula ósea, los cuales se fijaron por 30 segundos en un fijador de citrato, formol y acetona, seguido por un lavado con agua del tubo por 30 segundos. Las técnicas empleadas fueron: 1. Reacción del ácido peryódico de Schiff (PAS). 2. Sudán negro B (SNB). 3. Peroxidasa (PX) (única técnica que no requiere fijación). 4. Cloroacetato esterasa (CAE). 5. Alfa naftil acetato esterasa con y sin fluoruro de sodio (ANAE y ANAE + NaF). 6. Esterasas dobles (ANAE + CAE). 7. Fosfatasa ácida (FAC). 8. Fosfatasa alcalina (FAL). La PX se contratincionó con Wright y las demás técnicas con azul de metileno al $1 \%$. 


\section{RESULTADOS}

Pacientes del Hospital Calderón Guardia: Las leucemias agudas representaron el $66 \%$ de los casos. El resto fueron leucemias crónicas o trastornos relacionados. Los tipos más frecuentes de leucemia o trastornos relacionados, en orden decreciente fueron: leucemia aguda no linfocítica (LANL) (18), leucemia aguda linfocítica (LAL) (13), leucemia crónica mieloide (LCM) (8) y síndromes mielodisplásicos (5) (SMD). La leucemia crónica linfocítica (LCL) y la policitemia vera (PV) tuvieron frecuencias parecidas ( 2 casos en cada una). En la LANL el mayor número de casos se presentó en personas mayores de 45 años y en la LAL en pacientes menores de 35 años. La LCM mostró una frecuencia parecida en todas las edades. En la LAL y en la LANL, hubo mayor número de hombres afectados y en la LCM, se presentó un mayor número de mujeres. Los principales hallazgos citogenéticos fueron:

1. LANL (Cuadro 1, Apéndice 1). Predominó el subtipo M1. En esos casos se presentó cromosoma Philadelphia en dos, junto con otras aberraciones numéricas y en el resto de los pacientes únicamente variaciones numéricas. Las aberraciones numéricas más frecuentes fueron las trisomías de los cromosomas 19 y 20. En un caso de LANL-M5b hubo una delección del cromosoma 11 en mosaico junto con una línea normal. En todos los pacientes el NM fue de 46. Los casos (4) a los que no se les pudo analizar el cariotipo tuvieron un NM de 46 (Cuadro 2, Apéndice 1). Entre los pacientes hubo mezcla de células con diferentes grados de ploidía. En las células con 46 cromosomas predominaron las seudodiploides.

2. LAL (Cuadro 1, Apéndice 1). Se presentaron dos casos con delecciones del cromosoma 6 , en uno de ellos en mosaico junto con una línea normal. Los cariotipos del resto de pacientes tuvieron sólo aberraciones numéricas. Las variaciones numéricas más frecuentes fueron la monosomía 14 y las trisomías de los cromosomas 12,19 y 20 . El NM fue de 46, menos en un caso con 47. Dos pacientes a los que no se les pudo analizar el cariotipo presentaron un NM de 46 y 49 (Cuadro 2, Apéndice 1).
3. LCM. (Cuadro 1, Apéndice 1). Todos tuvieron cromosoma Philadelphia y sólamente en un caso ésa fue la única aberración. El resto mostraron más de un cromosoma Philadelphia junto con variaciones numéricas. Las más frecuentes fueron : monosomía del cromosoma 18 y trisomías de los cromosomas $15,16,19,20$ y 22 . El NM predominante fue de 46 cromosomas. Sólo un paciente tuvo un NM de 47.

4. Hubo un caso de PV en el cual la única aberración cromosómica fue la pérdida del cromosoma Y y un paciente con LCL en el cual se detectó una delección del cromosoma 1 y otra del cromosoma 6 y trisomías de los cromosomas 12.y 22. Su NM fue de 46 (Cuadro 1, Apéndice 1 ).

5. A ocho pacientes no se les pudo realizar estudio citogenético completo por fracaso de los cultivos celulares, hipocelularidad o cariotipos insuficientes para el análisis (Cuadro 2, Apéndice 1).

Hospital Nacional de Niños: Las leucemias agudas representaron el $98 \%$ de los casos. Las clases más frecuentes de leucemia fueron en orden decreciente: LAL (70\%) y LANL (27\%). El mayor número de casos de LAL se presentaron entre los tres y nueve años de edad. Dentro de la LAL, el subtipo LAL-1 (84\%) fue prevalente sobre el LAL-2 (16\%). No se presentó ningún caso de L3. En la LAL el $85 \%$ de las leucemias fueron del tipo B y más frecuentes en mujeres; mientras que el $15 \%$ fueron de tipo $\mathrm{T}$ y predominaron en hombres.

Las subclases más numerosas de LANL fueron en orden decreciente: M5a, M3, M4 y M5b. En la LANL el mayor número de casos se presentaron en niños menores de cuatro años y en la LAL en niños menores de siete años. En la LANL la distribución por sexos fue similar y en la LAL hubo un ligero exceso de varones afectados, aunque la diferencia no fue significativa. Se presentaron seis casos (5.6\%) de leucemia en niños menores de un año. Entre ellos hubo un número similar de leucemias agudas linfoides y mieloides. fueron: 
1. LAL-1 (Cuadro 3, Apéndice 1). Se presentó una delección del cromosoma 5, una delección del cromosoma 6 , una translocación entre los cromosomas 8 y 14, un isocromosoma 17 y una inversión del cromosoma 11. En todos esos casos además se detectaron variaciones numéricas. El resto de los pacientes mostraron solamente aberraciones numéricas. Las más importantes fueron las trisomías de los cromosomas 8, 19, 20, 21 y 22. Prevaleció el NM de 46 cromosomas, aunque un caso presentó 57 y otro 44 cromosomas. De cinco pacientes a los que no fue posible analizar el cariotipo cuatro tuvieron un número modal de cromosomas de 46 y el otro mayor de 50 (Cuadro 4, Apéndice 1).

2. LAL-2 (Cuadro 3, Apéndice 1). Se detectó un isocromosoma 17 y una delección del cromosoma 6. El resto de los pacientes mostraron sólo aberraciones numéricas. Dentro de las fluctuaciones numéricas las más frecuentes fueron las trisomías de los cromosomas $20 \mathrm{y}$ 21. En todos los casos el NM fue de 46, exceptuando uno al cual no se le pudo analizar el cariotipo y el cual mostró un NM mayor de 60 .

3. Dentro de los pacientes con LAL y número modal de 46, en aproximadamente la mitad de los casos se presentó una mezcla de células con diferentes ploidías: $46,<46$ y >46 cromosomas. Sin embargo se presentaron otras combinaciones de ploidías con menores frecuencias. Dentro de las células con 46 cromosomas en la mayoría de los casos fueron seudodiploides y en el resto una mezcla de células normales y seudodiploides.

4. LANL (Cuadro 3, Apéndice 1). La mayoría de pacientes mostraron sólo aberraciones numéricas. En un caso se detectó material adicional en el cromosoma 2 de origen desconocido y en otro una delección del cromosoma 11. Hubo un paciente con Síndrome de Down, el cual presentó LAM-M5a. Las variaciones numéricas más frecuentes fueron: monosomías de los cromosomas $15,17,21$ y 22 y trisomías del 12, 17, 19. En esos pacientes el NM fue de 46. Varios casos a los cuales no se les pudo analizar el cariotipo mostraron NM de 46, 47 y 57 (Cuadro 4, Apéndice 1).
5. Hubo dos pacientes con SMD en los cuales sólo se encontraron aberraciones numéricas. Las más frecuentes fueron las trisomías de los cromosomas 19,20 y 22 y presentaron un NM de 46 (Cuadro 3, Apéndice 1).

6. A diez pacientes no se les pudo realizar estudio citogenético completo porque fracasaron los cultivos celulares, hipocelularidad o cariotipos insuficientes para el análisis (Cuadro 4, Apéndice 1).

\section{DISCUSION}

Las leucemias agudas representaron la gran mayoría de los casos estudiados. Al igual que se ha informado en otros estudios, las leucemias agudas linfocíticas fueron más frecuentes en personas menores de 15 años (Heim y Mitelman 1987).

En niños la L1 es el tipo más común, como se detectó en nuestro estudio. La L2 es rara en niños y más común en adultos (Boros y Bennett 1990). Los porcentajes de los subtipos L1 y L2 de LAL encontrados en esta investigación, fueron semejantes a los informados según la clasificación FAB (Miller et al. 1981).

En aproximadamente el $90 \%$ de los casos de LAL se pueden detectar anormalidades cromosómicas (Pui et al. 1990). Nosotros sólo detectamos un paciente con cariotipo normal.

El cromosoma 5 ha sido implicado en la leucemogénesis de los adultos pero sólo raramente en la leucemia infantil (Mayer et al. 1995). En algunos casos raros de LAL se puede presentar $5 \mathrm{q}-$. La delección ha sido siempre interpretada como intersticial y su extensión varía (Sandberg 1990). En nuestro estudio se presentó 5q- en un niño con LAL, cuya extensión no pudo ser precisada.

La delección del brazo largo del cromosoma 6 fue señalada desde 1976, como un evento cariotípico recurrente por Oshimura y Sandberg. 6q- es relativamente común en la LAL y ocurre en el 5-10\% de los casos con anormalidades clonales. Globalmente, 6q- se considera un marcador confiable para las malignidades linfoides (Heim y Mitelman 1987), como ocurrió en nuestros casos. 
Las translocaciones cromosómicas recurrentes son relativamente comunes en ciertos neoplasmas, particularmente las leucemias , linfomas y algunos sarcomas (Heim y Mitelman 1987). En nuestro estudio se detectaron dos tipos de translocaciones: $t(8 ; 22$ )(q24;q11) en un paciente con LLA-1 B y la $\mathrm{t}(9 ; 22)$ (la cual será discutida más adelante). La t(8;22), junto con otras dos, en las que participa el cromosoma 8 en intercambio recíproco de segmentos con el 14 y 2 , constituyen un grupo de translocaciones notablemente asociadas con neoplasias de células B. Se les detecta tanto en leucemias como linfomas (especialmente Burkitt) (Berger y Bernheim 1982). La LAL asociada con estas translocaciones tiene casi siempre morfología L3 y sólo ocasionalmente morfología L1 o L2 (Heim y Mitelman 1987).

El i(17q) es el isocromosoma más común que ocurre en las neoplasias humanas. Su alta incidencia en las enfermedades mieloides está bien establecida (Sandberg 1990). Sin embargo, se han informado casos de LAL en los que se presenta como la única anormalidad o acompañado por otras aberraciones (Peetre et al. 1987). En nuestro estudio se encontró en dos niños con LAL.

Las alteraciones en la ploidía, son comunes en la leucemia aguda linfocítica y tienen significado pronóstico (Secker-Walker 1990). La hiperdiploidía (mayor de 50 cromosomas por célula) está asociada con un pronóstico mejor que el usual (Kaspers et al. 1995). La seudodiploidía está ligada a un pronóstico más pobre (Pui et al. 1990), mientras que la hipodiploidía tiene un pronóstico mucho peor que el usual (Pui et al. 1987). Dentro de nuestros pacientes hubo tres niños con LAL con más de 50 cromosomas y dos niños con hipodiploidía.

Las anormalidades cromosómicas son muy frecuentes en las leucemias agudas mieloides de novo y un impacto pronóstico independiente ha sido asignado, a los cambios recurrentes más comunes (Dastugue et al. 1995).

En nuestra investigación se detectaron dos casos de del (11)(q23), uno en un niño y otro en un adulto, ambos M5b. Varios rearreglos dife- rentes de 11q, que involucran las bandas q23 y q24, han sido asociados con LANL. La mayoría son translocaciones y delecciones (Kaneko et al. 1986). Las anormalidades de 11q están claramente correlacionadas con el subtipo M5 y se observan en cerca del 35\% de todos los pacientes con LANL-M5 (Berger et al. 1982).

El tipo M1 de la LANL continúa siendo un conglomerado de leucemias agudas bastante pobremente definido. Este grupo también contiene un alto porcentaje de leucemias secundarias. La incidencia de cromosoma Philadelphia $(\mathrm{Ph})$ en la LANL M1 es alrededor del $18 \%$, mientras que en todos los casos de LANL es de cerca 5\% (Sandberg 1990). Entre nuestros pacientes adultos la mayoría fueron del tipo M1 y dos de los casos presentaron cromosoma $\mathrm{Ph}$.

En la leucemia crónica mieloide, al menos el $95 \%$ de los casos, están caracterizados por la presencia de una translocación recíproca entre los cromosomas 9 y 22 (Colleoni et al. 1996). En aproximadamente el $80 \%$ de ellos la progresión a la fase acelerada y blástica es precedida o acompañada por anormalidades cromosómicas recurrentes adicionales (Anónimo 1978) como un cromosoma Philadelphia extra, un i(17q), trisomía 8 y trisomía 19. En nuestra investigación sólo un paciente presentó la $\mathrm{t}(9 ; 22)$ como única anormalidad. El resto mostraron otras aberraciones cromosómicas junto con otro cromosoma $\mathrm{Ph}$.

La leucemia crónica linfocítica es con mayor frecuencia una enfermedad asociada con la edad media o vejez. La más frecuente es la $\mathrm{B}$ (Glassman 1997). Algunas de las aberraciones cromosómicas encontradas con frecuencia en LCL-B son +12 y del(6q) (Glassman 1997). Uno de nuestros pacientes con LCL presentó esas dos anormalidades, al lado de otras.

En los síndromes mielodisplásicos algunas aberraciones cromosómicas muestran una gran asociación, como 5q-, $-5,-7,+8$, y delecciones y translocaciones de 11q y $12 p$ (Heim y Mitelman 1987). Entre nuestros pacientes se presentaron dos niños con SMD, los cuales únicamente mostraron aberraciones numéricas, y dentro de ellas sólo +8 estuvo dentro de la lista de aberraciones frecuentes publicadas. 
Se detectó un único caso de policitemia vera (PV) en un paciente de 79 años. Una proporción de estos pacientes presentan anormalidades cromosómicas clonales al diagnóstico (cerca del 15\%) (Heim y Mitelman 1987). La única anormalidad que se encontró en este paciente fue la pérdida del cromosoma $Y$, que bien puede ser un fenómeno relacionado con su edad avanzada, sin importancia patogenética, ya que el cariotipo constitucional también mostró tal anormalidad.

Esta investigación representó la primera efectuada en nuestro país, en la cual se realizó un estudio citoquímico detallado, junto con un estudio citogenético. Esto permitió una clasificación confiable del tipo de leucemia y la posterior comparación de los datos citogenéticos con los datos de la literatura, con los cuales hubo una gran coincidencia.

\section{AGRADECIMIENTOS}

Agradecemos a la Fundación Santiago Crespo y a la Caja Costarricense del Seguro Social, por la donación de equipo para la realización de esta investigación. Asímismo a la Organización Panamericana de la Salud y a la Vicerrectoría de Investigación, de la Universidad de Costa Rica, por el soporte económico brindado.

\section{RESUMEN}

Se realizó estudio citogenético y/o citoquímico a 166 pacientes con leucemia o trastomos relacionados, referidos a dos hospitales costarricenses (HNN y HCG). En los enviados al HCG (mayores o iguales a 14 años), las leucemias agudas representaron el $66 \%$ de los casos. Entre ellos los tipos de trastormos más frecuentes fueron en orden decreciente: LANL (>M1), LAL, LCM (entre ellos todos mostraron cromosoma Ph) y SMD. Dentro de los casos enviados al HNN ( $<14$ años) las leucemias agudas constituyeron el $98 \%$ de los casos. En éstos las clases más frecuentes fueron en orden decreciente: LAL (70\%): LAL-1 $(84 \%)$ y LAL-2(16\%) y la LANL (27\%): M5a $>$ M3 $>$ M4 $>$ M5b. En la LAL el $85 \%$ fueron de tipo B y predominaron en mujeres mientras que el $15 \%$ de tipo $\mathrm{T}$ y más frecuentes en varones. Hubo un $5.6 \%$ de leucemia infantil, la cual presentó un número similar de agudas linfoides y mieloides. Los hallazgos citogenéticos fueron bastante parecidos a los publicados en otros países tropicales y templados.

\section{REFERENCIAS}

Anónimo. 1978. Chromosomes in $\mathrm{Ph}+$ chronic granulocytic leukemia. Br. J. Haematol. 39:305-309.

Anónimo. 1981. Cancer Genet. Cytogenet. 4:95-142

Anónimo. 1992. Guidelenes for cancer cytogenetics. Supplement to An International System for Human Cytogenetic Nomenclature. Karger, Basilea, Suiza, 54p.

Berger, R., A. Bernheim \& A. de la Chapelle. 1982. Chromosome rearrangements in acquired malignant diseases. Cytogenet. Cell Genet. 32:205-207.

Berger, R. \& A. Bernheim. 1982. Cytogenetic studies on Burkitt's lymphoma-leukemia. Cancer Genet. Cytogenet. 7:231-244.

Boros L. \& J.M. Bennett. 1990. Morphology and cytochemistry. p.361- 382. In: E.S Henderson. \& T.A. Lister (eds.). William Dameshek and Frederick Gunz's leukemia. W.B. Saunders, Filadelfia, Pensilvania.

Colleoni, G.W., F.F. Costa , C.R. Grignolli, R.S. Silva, M.L. Chauffaille, J. Kerbauy \& S.T. Saad. 1996. Prognostic significance of BCR-ABL rearrangement in çhronic myeloid leukemia. Braz. J. Med. Biol. Res. 29:1307-1310.

Dacie, J.V. \& S.M. Lewis. 1987. Citoquímica de las células sanguíneas y técnicas suplementarias, p.92-101. In Hematología práctica. Toray, Barcelona.

Dastugue, N., C. Payen, M. Lafage-Pochitaloff, P. Bernard, D. Leroux, F. Huguet-Rigal, A.M. Stoppa, G. Marit, L. Molina, M. Michallet, D. Maraninchi, M. Attal \& J. Reiffers. 1995. Prognostic significance of karyotype in de novo adult acute myeloid leukemia. Leukemia 9:1491-1498.

Glassman, A. 1997. Cytogenetics. An evolving role in the diagnosis and treatment of cancer. Clin. Lab. Med. 17:21-37.

Heim, S. \& F. Mitelman. 1987. Cancer Cytogenetics. Alan R.Liss, Nueva York. 309p.

Kaneko, Y., N. Maseki, N. Takasaki, M. Sakurai, Y. Hayashi, S. Nakazawa, T. Mori, M. Sakurai, T. Takeda, T. Shikano \& Y. Hiyoshi. 1986. Clinical and hematologic characteristics in acute leukemia with 11 q23 translocations. Blood 67:484-491. 
Kaspers, G.J., L.A. Smets, R. Pieters, C.H. Vanzantwijk, E.R. Vanwering \& A.J. Veerman. 1995. Favorable prognosis of hyperdiploid common acute lymphoblastic leukemia may be explained by sensitivity to antimetabolites and other drugs: Results of an in vitro study. Blood 85:751-756.

Le Beau, M. 1991. Cytogenetic analysis of hematological malignant diseases. p.395-445. In M.J. Barch (ed). The ACT Cytogenetics Laboratory Manual. Raven, Nueva York.

Mayer, J.L., M.R. Seashore \& F.M. Hajjar. 1995. Translocation $(5 ; 6)$ associated with spontaneously remitting congenital leukemia. Canc. Genet. Cytogenet. 81:38-41.

Miller, D.R., S. Leikin, V. Albo, H. Sather \& D. Hammond. 1981. Prognostic importance of morfhology (FAB classification) in childhood acute lymphoblastic leukaemia (ALL). Br. J. Haematol. 48:199-206.

Oshimura, M. \& A.A. Sandberg. 1976. Chromosomal 6qanomaly in acute lymphoblastic leukaemia. Lancet ii:1045-1046.

Peetre, C., P.G. Nilsson \& F. Mitelman. 1987. Isochromosome $17 \mathrm{q}$ in a patient with acute myeloblas c leukemia. Cancer Genet. Cytogenet. 24:315-318.

Pui, C.H., D.L. Williams, S.C. Raimondi, G.K. Rivera, A.T. Look, R.K. Dodge, S.L. George, F.G. Behm, W.M. Crist \& S.B. Murphy. 1987. Hypodiploidy is associated with a poor prognosis in childhood acute lymphoblastic leukemia. Blood 70:247-253.
Pui, C.H., W.M. Crist \& A.T. Look. 1990. Biology and clinical significance of cytogenetic abnormalities in childhood acute lymphoblastic leukemia. Blood 76:1449-1463.

Rooney, D.E. \& B.H. Czepulkowski. 1986. Human Cytogenetics : A practical approach. OIRL, Oxford. 241p.

Sandberg, A.A. 1990. The Chromosomes in Human Cancer and Leukemia. Elsevier, Nueva York, 1315p.

Scott, C.S., G.J. Den Ottolander, D. Swirsky, G.A. Pangalis, J.L. Vives Corrons, A.D. Pasquale, L. Van Hove, J.M. Bennett, K. Namba, G. Flandrin, S.M. Lewis \& I.A. Polliack. 1993. Recommended procedures for the clasification of acute leukaemias. Leuk.\& Lymph. 11:37-50.

Seabright, M. 1971. A rapid banding technique for human chromosomes. Lancet 2: 971-972.

Secker-Walker, L.M. 1990. Prognostic and biological importance of chromosome findings in acute lymphoblastic leukemia. Cancer Genet. Cytogenet. 49:1-13.

Williams, D.L., H. Harris, K.J. Williams, M.J. Brosius \& W. Lemonds. 1984. A direct bone marrow chromosome technique for acute lymphoblastic leukemia. Cancer Genet. Cytogenet. 13:239. 


\section{APENDICE 1}

\section{CUADRO 1}

Edad, sexo, tipo de trastorno hematológico, cariotipo compuesto (cp) y número modal de cromosomas (NM), de pacientes reclutados en el Hospital Dr. Calderón Guardia.

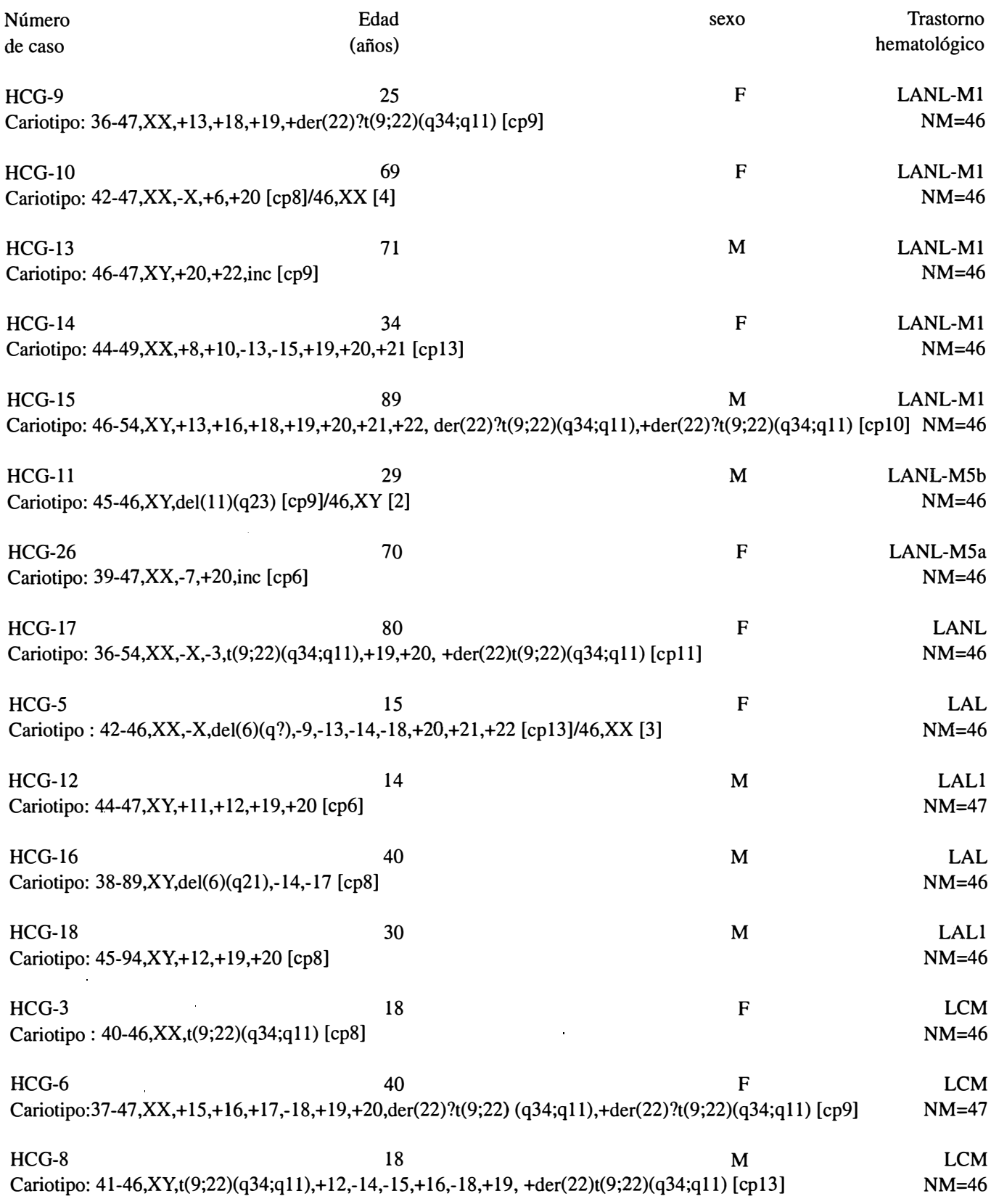




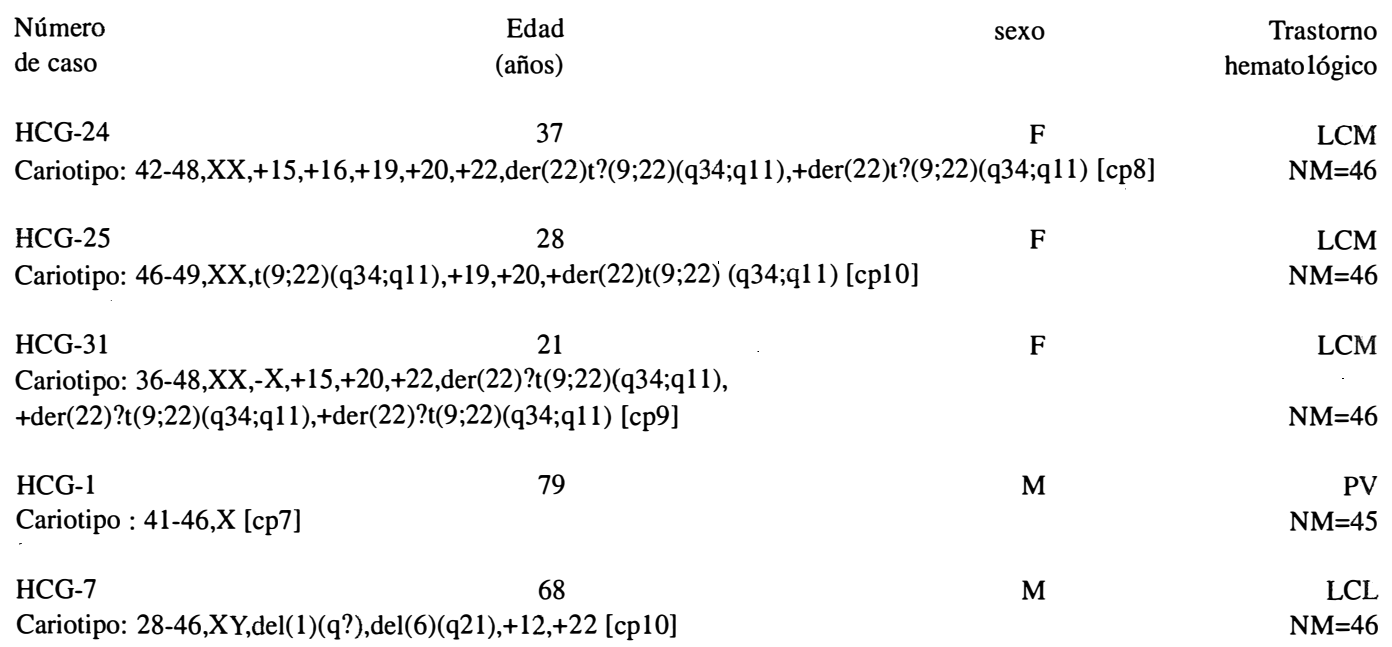

\section{CUADRO 2}

Edad, sexo, rango cromosómico (RC), número modal de cromosomas (NM) y tipo de trastorno hematológico, en pacientes reclutados en el Hospital Dr. Calderón Guardia.

$\begin{array}{lrccrr}\begin{array}{l}\text { Número } \\ \text { de caso }\end{array} & \begin{array}{r}\text { Edad } \\ \text { (años) }\end{array} & \text { Sexo } & \text { RC } & \text { NM } & \begin{array}{r}\text { Trastorno } \\ \text { hematológico }\end{array} \\ \text { HCG-2 } & 15 & \text { M } & 42-92 & 46 & \text { LANL-M1 } \\ \text { HCG-19 } & 50 & \text { F } & 40-47 & 46 & \text { LANL-M3 } \\ \text { HCG-21 } & 30 & \text { M } & 40-47 & 46 & \text { LANL } \\ \text { HCG-29 } & 36 & \text { M } & 28-56 & 46 & \text { LANL-M5a } \\ \text { HCG-4 } & 71 & \text { F } & 47-92 & 49 & \text { LAL2 } \\ \text { HCG-27 } & 28 & \text { F } & 41-50 & 46 & \text { LAL1 } \\ \text { HCG-23 } & 75 & \text { F } & 32-50 & 46 & \text { LCL } \\ \text { HCG-28 } & 59 & \text { F } & 32-46 & 46 & \text { LCM }\end{array}$

\section{CUADRO 3}

Edad, sexo, tipo de trastorno hematológico, cariotipo compuesto (cp) y número modal de cromosomas (NM) de pacientes reclutados en el Hospital Nacional de Niños.

\begin{tabular}{|c|c|c|c|}
\hline $\begin{array}{l}\text { Número } \\
\text { de caso }\end{array}$ & $\begin{array}{r}\text { Edad } \\
\text { (años.meses) }\end{array}$ & sexo & $\begin{array}{l}\text { Tipo de trastorno } \\
\text { hematológico }\end{array}$ \\
\hline $\begin{array}{l}\mathrm{HNN}-1 \\
\text { Cariotip }\end{array}$ & $\mathrm{Y}[2]$ & $\mathbf{M}$ & $\begin{array}{r}\text { LAL1-B } \\
\mathrm{NM}=46\end{array}$ \\
\hline $\begin{array}{l}\text { HNN-3 } \\
\text { Cariotipc }\end{array}$ & 20 [cp9] & $\mathrm{F}$ & $\begin{array}{r}\text { LAL1-B } \\
\mathrm{NM}=46\end{array}$ \\
\hline
\end{tabular}




\begin{tabular}{|c|c|c|}
\hline $\begin{array}{r}\text { Edad } \\
\text { (años.meses) }\end{array}$ & sexo & $\begin{array}{l}\text { Tipo de trastorno } \\
\text { hematológico }\end{array}$ \\
\hline $\begin{array}{l}\text { HNN-4 } \\
\text { Cariotipo: 46,XX,inc [9] }\end{array}$ & $\mathrm{F}$ & $\begin{array}{r}\text { LAL1-B } \\
\mathrm{NM}=46\end{array}$ \\
\hline $\begin{array}{l}\text { HNN-5 } \\
\text { Cariotipo: 45-49,XY,del(6)(q2?) [cp6] }\end{array}$ & $\mathbf{M}$ & $\begin{array}{r}\text { LAL1-B } \\
\mathrm{NM}=57\end{array}$ \\
\hline $\begin{array}{l}\text { HNN-9 } \\
\text { Cariotipo: } 44-47, \mathrm{XY},-2,-3,+8, t(8 ; 22)(\mathrm{q} 24 ; \mathrm{q} 11),-14 \text { [cp12] }\end{array}$ & $\mathbf{M}$ & $\begin{array}{r}\text { LAL1-B } \\
\mathrm{NM}=46\end{array}$ \\
\hline $\begin{array}{l}\text { HNN-13 } \\
\text { Cariotipo: } 45-54, X Y,+20,+21,+22[\text { cp5] }\end{array}$ & $\mathbf{M}$ & $\begin{array}{r}\text { LAL1-B } \\
\mathrm{NM}=46\end{array}$ \\
\hline $\begin{array}{l}\text { HNN-16 } \\
\text { Cariotipo: } 47-50, X Y,+11,+19[\text { cp4] }\end{array}$ & $\mathbf{M}$ & $\begin{array}{r}\text { LAL1-B } \\
\mathrm{NM}=44\end{array}$ \\
\hline $\begin{array}{l}\text { HNN-17 } \\
\text { Cariotipo: } 42-49, \mathrm{XX},+13,-17,+\mathrm{i}(17)(\mathrm{q} 11.1),+19,+20,+21,+22[\mathrm{cp} 17]\end{array}$ & $\mathrm{F}$ & $\begin{array}{r}\text { LAL1-B } \\
\mathrm{NM}=46\end{array}$ \\
\hline $\begin{array}{l}\text { HNN-35 } \\
\text { Cariotipo: 46-91,XY,+21,inc [cp6] }\end{array}$ & $\mathrm{M}$ & $\begin{array}{r}\text { LAL1-B } \\
\mathrm{NM}=46\end{array}$ \\
\hline $\begin{array}{l}\text { HNN-50 } \\
\text { Cariotipo: } 42-46, \mathrm{XY}, \operatorname{inv}(11)(\mathrm{q} 13.1 \mathrm{q} 21),+19,+20[\mathrm{cp} 8]\end{array}$ & $\mathbf{M}$ & $\begin{array}{r}\text { LAL1-B } \\
\mathrm{NM}=46\end{array}$ \\
\hline $\begin{array}{l}\text { HNN-32 } \\
\text { Cariotipo: } 46, X X,+1, \text { inc [cp9] }\end{array}$ & $\mathrm{F}$ & $\begin{array}{r}\text { LAL1-B } \\
\mathrm{NM}=46\end{array}$ \\
\hline $\begin{array}{l}\text { HNN-11 } \\
\text { Cariotipo: 44-45,XY,+16;+21 [cp10]/46,XY [5] }\end{array}$ & $\mathbf{M}$ & $\begin{array}{r}\text { LAL2-B } \\
\mathrm{NM}=46\end{array}$ \\
\hline $\begin{array}{l}\text { HNN-40 } \\
\text { Cariotipo: } 40-47, \mathrm{XX},-17, \mathrm{i}(17)(\mathrm{q} 11.1),+19,+20,+21,+22[\mathrm{cp} 13]\end{array}$ & $\mathrm{F}$ & $\begin{array}{l}\text { LAL2-T } \\
\mathrm{NM}=46\end{array}$ \\
\hline $\begin{array}{l}11.9 \\
\text { HNN-41 } \\
\text { Cariotipo: } 43-47, \mathrm{XX}, \operatorname{del}(6)(\mathrm{q} ? 25),-8,-9,+12,+13,+14,+20,+21 \text { [cp14] }\end{array}$ & $\mathrm{F}$ & $\begin{array}{l}\text { LAL2-B } \\
\mathrm{NM}=46\end{array}$ \\
\hline $\begin{array}{l}\text { HNN-44 } \\
\text { Cariotipo: } 46, X X \text {,inc }\end{array}$ & $\mathrm{F}$ & $\begin{array}{l}\text { LAL2-B } \\
\mathrm{NM}=46\end{array}$ \\
\hline $\begin{array}{l}\text { HNN-18 } \\
\text { Cariotipo: } 34-47, X X,-6,-9,-10,+13,-15,+19,-22[\operatorname{cp} 19] / 46, X X[3]\end{array}$ & F & $\begin{array}{r}\text { LANL } \\
\mathrm{NM}=46\end{array}$ \\
\hline $\begin{array}{l}\text { HNN-2 } \\
\text { Cariotipo: } 42-46, \mathrm{XX},+12,+14,-17,+19[\mathrm{cp} 8]\end{array}$ & $\mathrm{F}$ & $\begin{array}{r}\text { LANL-M4 } \\
\mathrm{NM}=46\end{array}$ \\
\hline $\begin{array}{l}\text { HNN-6 } \\
\text { Cariotipo: } 46-3 \\
\text { Ch, XX, }+17,+18,+19,+21[\operatorname{cp} 11] / 46, \mathrm{XX}[4]\end{array}$ & $\mathrm{F}$ & $\begin{array}{r}\text { LANL-M3 } \\
\mathrm{NM}=46\end{array}$ \\
\hline $\begin{array}{l}\text { HNN-33 } 3.2 \\
\text { Cariotipo: } 36-47, \mathrm{XX},-1,+12,-13,-14,-16,+21 \mathrm{c},+22,-22[\mathrm{cp} 12] / 47, \mathrm{XX},+21 \mathrm{c}[2]\end{array}$ & $\mathrm{F}$ & $\begin{array}{r}\text { LANL-M5a } \\
\mathrm{NM}=46\end{array}$ \\
\hline $\begin{array}{l}\text { HNN-38 } \\
\text { Cariotipo: 46,XX, add(2)(q37.3) [6]/39-47,XX,add(2)(q37.3),+17,-21 [cp6] }\end{array}$ & $F$ & $\begin{array}{r}\text { LANL-M5a } \\
\mathrm{NM}=46\end{array}$ \\
\hline
\end{tabular}




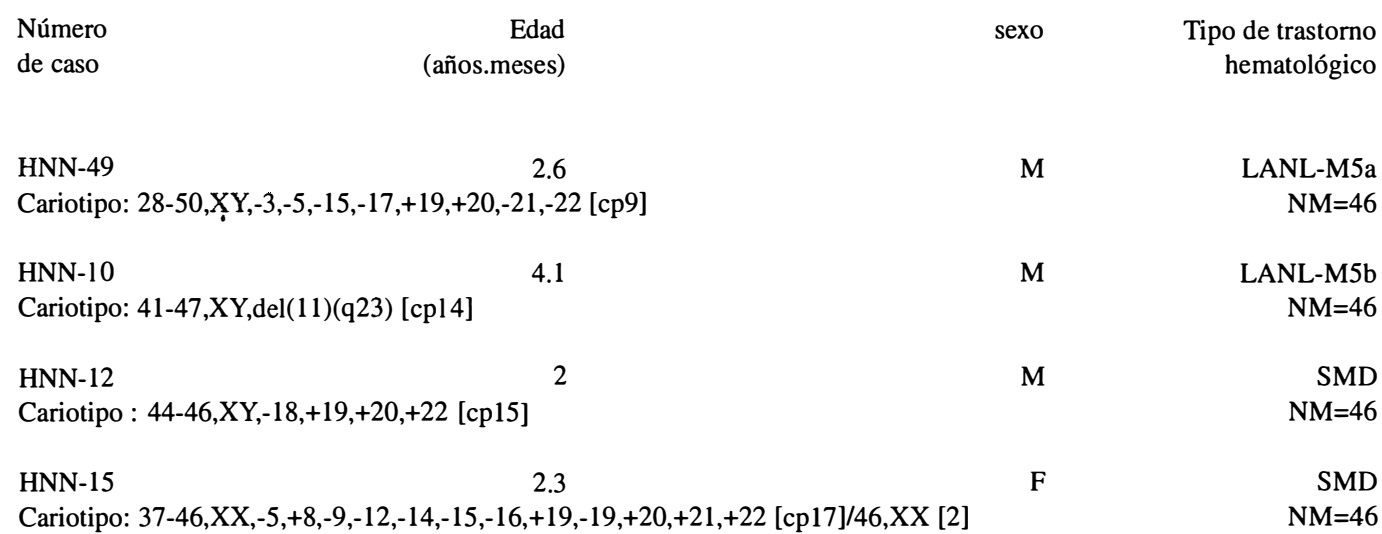

\section{CUADRO 4}

Edad, sexo, rango cromosómico $(R C)$, número modal de cromosomas $(N M)$ y tipo de trastorno hematológico, en pacientes reclutados en el Hospital Nacional de Niños.

$\begin{array}{lrrrrr}\begin{array}{l}\text { Número } \\ \text { de caso }\end{array} & \begin{array}{r}\text { Edad } \\ \text { (años) }\end{array} & \text { Sexo } & \text { RC } & \text { NM } & \begin{array}{r}\text { Trastorno } \\ \text { hematológico }\end{array} \\ \text { HNN-7 } & 6.2 & \text { M } & 47-52 & >50 & \text { LAL1-T } \\ \text { HNN-14 } & 1.3 & \mathrm{~F} & 46-117 & >60 & \text { LAL1-T } \\ \text { HNN-23 } & 5.2 & \mathrm{M} & 35-47 & 46 & \text { LAL1-B } \\ \text { HNN-28 } & 11.7 & \mathrm{M} & 32-46 & 46 & \text { LAL1-T } \\ \text { HNN-42 } & 12.4 & \mathrm{~F} & 41-55 & 46 & \text { LAL1-B } \\ \text { HNN-45 } & 9.5 & \mathrm{~F} & 40-61 & 46 & \text { LAL1-B } \\ \text { HNN-46 } & 6.8 & \mathrm{M} & 36-47 & 44 & \text { LAL-LCMJ } \\ \text { HNN-29 } & 14 \text { días } & \mathrm{M} & 41-49 & 46 & \text { LANL-M5a } \\ \text { HNN-31 } & 2.1 & \mathrm{~F} & 30-48 & 47 & \text { LANL-M5a } \\ \text { HNN-47 } & 0.11 & \mathrm{M} & 46-57 & 57 & \text { LANL-M4 }\end{array}$

